\title{
Structural aspects of human lactoferrin in the iron-binding process studied by molecular dynamics and small-angle neutron scattering ${ }^{\star}$
}

\author{
Lilia Anghel ${ }^{1, a}$, Aurel Radulescu ${ }^{2}$, and Raul Victor Erhan ${ }^{3,4, b}$ \\ 1 Institute of Chemistry (ICh), Academiei 3, MD-2028 Chisinau, Republic of Moldova \\ 2 Jülich Centre for Neutron Science (JCNS) at Heinz Maier-Leibnitz Zentrum (MLZ), Forschungszentrum Jülich GmbH, \\ Lichtenbergstraße 1, 85748 Garching, Germany \\ 3 Neutron materials characterization (NØYTRON), Institute for Energy Technology (IFE), Instituttveien 18, P.O. Box 40, \\ 2027 Kjeller, Norway \\ 4 Horia Hulubei National Institute for R\&D in Physics and Nuclear Engineering (IFIN-HH), Reactorului 30, P.O. Box MG-6, \\ Bucharest - Magurele, Romania
}

\begin{abstract}
Received 12 April 2018 and Received in final form 30 July 2018
Published online: 20 September 2018

(C) The Author(s) 2018, corrected publication November 2018. This article is published with open access at Springerlink.com
\end{abstract}

\begin{abstract}
Lactoferrin is a non-heme protein known for its ability to bind tightly Fe(III) ions in various physiological environments. Due to this feature lactoferrin plays an important role in the processes of iron regulation at the cellular level preventing the body from damages produced by high levels of free iron ions. The X-ray crystal structure of human lactoferrin shows that the iron-binding process leads to conformational changes within the protein structure. The present study was addressed to conformation stability of human lactoferrin in solution. Using molecular dynamics simulations, it was shown that Arg121 is the key amino acid in the stabilization of the Fe(III) ion in the N-lobe of human lactoferrin. The smallangle neutron scattering method allowed us to detect the structural differences between the open and closed conformation of human lactoferrin in solution. Our results indicate that the radius of gyration of apolactoferrin appears to be smaller than that of the hololactoferrin, $R_{g}=24.16( \pm 0.707) \AA$ and $R_{g}=$ $26.20( \pm 1.191) \AA$, respectively. The low-resolution three-dimensional models computed for both forms of human lactoferrin in solution also show visible differences, both having a more compact conformation compared to the high-resolution structure.
\end{abstract}

\section{Introduction}

Transferrins are a family of proteins that is responsible for regulating iron levels in living organisms. Subsequent studies have shown that these proteins in addition to $\mathrm{Fe}$ (III) ions are also able to bind a variety of other metals. As a route of delivery of such metals to living cells, the transferrin family plays an important role in living microorganism, since some metals are essential for many biological processes such as catalysis, photosynthesis, water oxidation, molecular reduction of oxygen and nitrogen fixation [1]. On the other hand, an increased cellular concentration of free metal ions causes deleterious effects associated with the increase of free oxidizing reac-

\footnotetext{
* The original version of this article was revised due to a retrospective Open Access order.

a e-mail: lilia.anghel@chem.asm.md

b e-mail: raul.erhan@ife.no
}

tive species concentration resulting in the damage of the cell components (proteins, lipids, DNA) or even apoptosis. Despite the biochemical function of protein, the reaction with metal ions induces conformational changes associated with various intermolecular interactions. Conformational rearrangements of large macromolecules are very difficult to study experimentally. A case in point is lactoferrin that changes its conformation from open (apolactoferrin) to closed (hololactoferrin) in the course of reaction with Fe(III) ions (see fig. 1) [2]. Therefore, the investigation of these intermolecular interactions using combined experimental and computational efforts will improve our understanding of these specific mechanisms at molecular level.

Lactoferrin is a glycoprotein with a molecular mass of about $75-80 \mathrm{kDa}$ [3]. This protein is highly conserved across the species [4] and its crystal structure consists of a single polypeptide chain of 600-700 amino acid residues. The chain is folded into two ellipsoidal lobes, referred to 
(a) Closed hololactoferrin
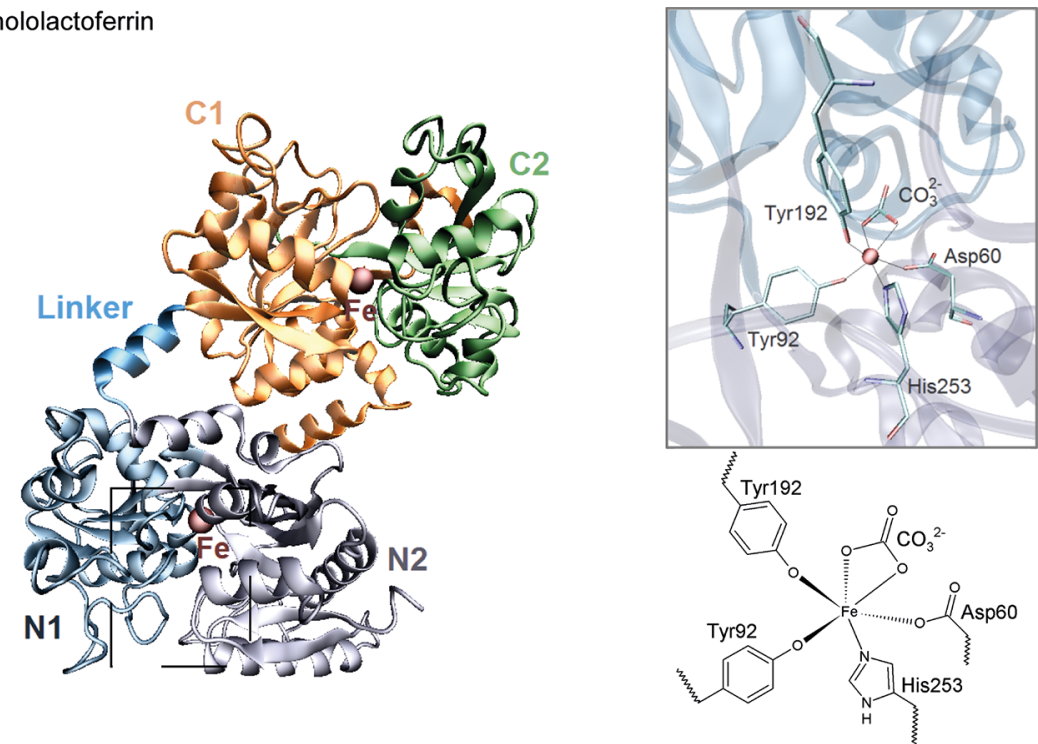

(b) Partially opened monoferric human lactoferrin
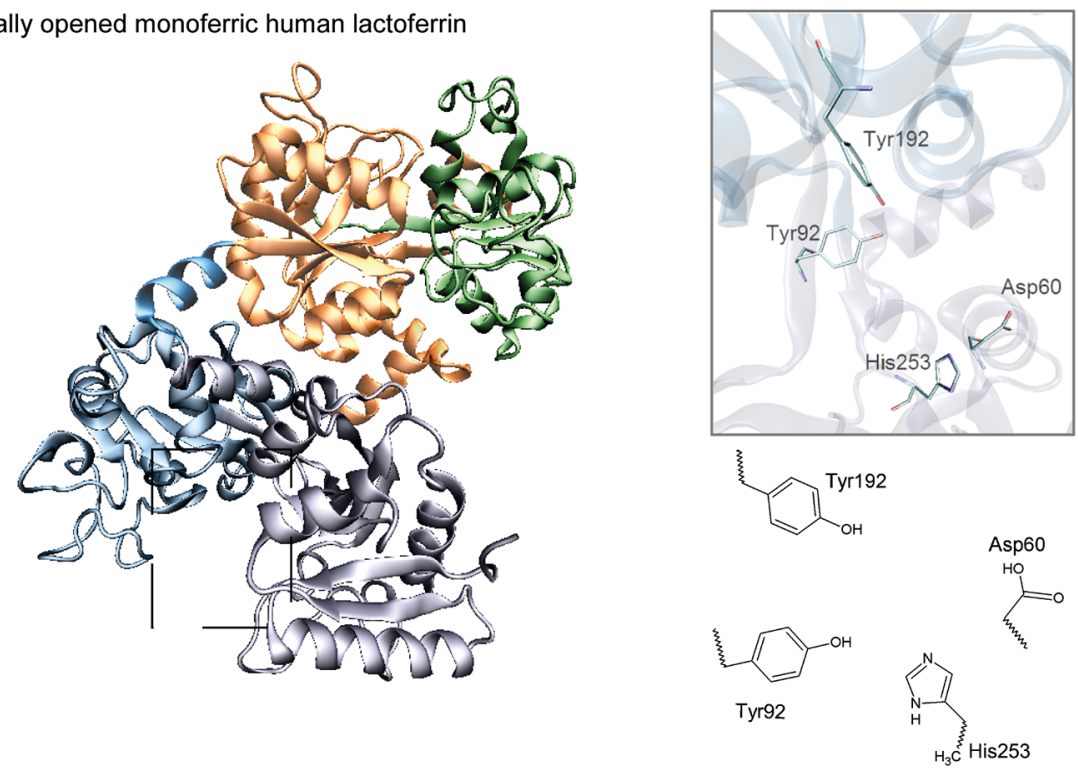

Fig. 1. Cartoon representation of the overall structure of (a) human hololactoferrin (PDB entry 1B0L) and (b) monoferric human lactoferrin (PDB entry 1LFH), ferric ions are represented as spheres. A close-up view of the metal ion coordination site in the N-lobe of human lactoferrin in the closed (a) and open form (b) with the residues Asp60, Tyr92, Tyr192, His253.

as $\mathrm{N}$ and $\mathrm{C}$ lobes that are connected by a helical peptide chain. Each lobe is further divided in two domains, N1 and $\mathrm{N} 2$ in the N-lobe and $\mathrm{C} 1$ and $\mathrm{C} 2$ in the C-lobe. An ironbinding site is available in each lobe where the metal ion is coordinated by four amino acid side-chains: a carboxylate from one aspartate residue, two phenolate oxygen atoms from two tyrosine residues and one imidazole from one histidine residue (fig. 1). The metal ion is stabilized in the protein-binding site through two oxygen atoms from a carbonate ion [5].

Lactoferrin has been the subject of study for many years as its biological functions are not completely established nor understood. Until now, reports can be found in the literature regarding its bacteriostatic [6], antiinflammatory [7], immunomodulatory [8], and even anticarcinogenic $[9,10]$ properties that mainly derive from the ability of binding Fe(III) ions. Experimental and theoretical studies have shown the complexity of the Fe(III)binding reaction, in the N-lobe of lactoferrin that is triggered by the change in the protonation state of the Tyr192 residue and involves the amino acid residues from the second coordination sphere Arg121, Arg210 and Lys301 [1114]. In view of the foregoing, the focus of our study was to assess the influence of Arg121, Arg210 on the conformation stability of human lactoferrin using molecular dynamics simulations. The conformation stability of human lactoferrin in solution was appraised using the small-angle neutron scattering (SANS) technique. The low-resolution three-dimensional models of human lactoferrin in the open and closed forms were computed. Our results include the estimation for the molecular size and conformation of both forms of human lactoferrin. 


\section{Materials and methods}

\subsection{Molecular dynamics simulations}

All molecular dynamics simulations were performed using Gromacs 4.5.5 software package $[15,16]$. Molecular dynamics simulations were conducted on four structures: open apolactoferrin (S1), closed apolactoferrin (S2), carbonate closed lactoferrin (S3), hololactoferrin (S4). To assess the importance of Arg121 and Arg210 residues in the iron binding and release, two systems were additionally created with the mutated forms of hololactoferrin R121 (S5) and R210 (S6).

The systems S5 and S6 were obtained by artificial mutation of Arg121 and Arg210 to Ala121 and Ala210, respectively, in the 1B0L.pdb file. The PDB Swiss Viewer software [17] was used for the reconstruction of the amino acids side-chain atoms that were missing.

The protonation states of residues in the proteins corresponded to the physiological $p \mathrm{H}$ conditions $(p \mathrm{H}$ 7.4). In all molecular dynamics simulation Tyr92, Tyr192, His253, Tyr435, Tyr528 and His597 were considered deprotonated. The OPLS-all atoms force field [18] was employed to build the topology of the protein. The atomic charges for the carbonate ions and the non-bonded parameters of $\mathrm{Fe}(\mathrm{III})$ ions were taken from the literature [19,20]. Periodic boundary conditions were applied in all directions using a cubic cell with a minimal distance between the protein and the wall of the cell set to $10 \AA$. Then, water molecules, modeled using the TIP3P function, were added to the system. Seven chloride ions were randomly placed in the box to neutralize the positively charged protein.

Once the system was built, the energy minimization step was applied to ensure that the system will not clash during the simulation. A $500 \mathrm{ps}$ equilibration was performed in the canonical thermodynamic (NVT) ensemble, where the temperature of the protein and the rest of the system were coupled to $300 \mathrm{~K}$ using the velocity rescaling algorithm Berendsen thermostat. This was followed by a 500 ps equilibration in the isothermal-isobaric thermodynamic ensemble (NPT). The Parrinello-Rahman barostat was used to equilibrate the systems at a normal pressure of 1 bar. The time step for the equilibration was set to $2 \mathrm{fs}$.

The positions of the protein and the rest of the system were constrained at this stage, and particle-mesh Ewald (PME) summations were used for long-range electrostatics and the van der Waals cut-off was set at $14 \AA$. The position restrains were released to produce the molecular dynamics simulation. The linear constraint solver (LINCS) algorithm was used to constraint bond lengths in the protein and an integration time step of $2 \mathrm{fs}$. A total run of $5 \mathrm{~ns}$ was conducted for each simulation. The subsequent data analysis was performed using Gromacs tools and VMD software [21].

\subsection{Protein sample preparation}

Diferric human lactoferrin purchased commercially (Sigma-Aldrich) was used in our studies. For UV-Vis spectroscopic measurements, the solutions of lactoferrin were prepared by dissolving the lyophilized powder in $50 \mathrm{mM}$ HEPES and $p \mathrm{H}$ was adjusted to 7.4 with $0.1 \mathrm{M} \mathrm{NaOH}$. The concentration of human lactoferrin was fixed at $2 \mathrm{mg} / \mathrm{mL}$. The lactoferrin solution was titrated with $1 \mathrm{mM}$ EDTA to obtain the open form. Milli-Q water was used in all sample preparations.

For SANS measurements, the lyophilized powder of human lactoferrin was dissolved in $50 \mathrm{mM}$ HEPES in $100 \% \mathrm{D}_{2} \mathrm{O}$. The total starting protein concentration was $5 \mathrm{mg} / \mathrm{mL}$. The apo-form of the protein was obtained by adding $100 \mu \mathrm{L}$ of $10 \mathrm{mM}$ EDTA solution to a volume of $900 \mu \mathrm{L}$ of protein solution.

\section{$2.3 \mathrm{UV}-\mathrm{Vis}$ spectroscopy}

UV-Vis absorption spectra were recorded in the range from 200 to $600 \mathrm{~nm}$ using a Perkin Elmer Lambda 25 Spectrophotometer equipped with $1.0 \mathrm{~cm}$ quartz cells at $298 \mathrm{~K}$.

\subsection{SANS measurements}

SANS investigation was carried out using the KWS-2 diffractometer, operated by the Jülich Centre for Neutron Science, Outstation at the Research Neutron Source Heinz Maier-Leibnitz FRM II, Garching, Germany [22]. The scattering spectra were recorded in two series, for $10 \mathrm{~min}$ and $60 \mathrm{~min}$ measurement time with a fixed neutron wavelength $\lambda=5.7 \AA$. The samples were held in $300 \mu \mathrm{L}$ quartz Helma cells, at a temperature of $293 \mathrm{~K}$. Data collected from two distances sample-to-detector (1.12 and $5.73 \mathrm{~m}$ ) were merged using the dedicated software QtiKWS to obtain a complete spectrum for each analyzed sample in the 0.007 to $0.4 \AA^{-1} q$-range. SANS data analysis and molecular reconstruction were performed using ATSAS 2.7.1 software package [23].

\section{Results and discussion}

\subsection{Molecular dynamics simulations}

\subsubsection{Conformation stability of human lactoferrin}

Molecular dynamics simulations were carried out to assess the stability of human lactoferrin in the four conformations: open apolactoferrin (S1), closed apolactoferrin (S2), iron-free closed form of lactoferrin (S3) and hololactoferrin (S4).

Structural deviations along the trajectories were evaluated using the root mean square deviations (RMSD) of the protein $\mathrm{C} \alpha$ atoms (see fig. 2(a)). In all four simulations, the RMSD steadily raises in the first $100 \mathrm{ps}$, reaching the typical value of 1.5-2.0 . Along the entire simulation, RMSD exhibits fluctuations that reach maximal values of 2.5-3.8 $\AA$ which vary for the four studied systems. As is shown in fig. 2(a), the S3 system has the least structure deviations, whilst the greatest deviations were registered for the S4 system. The RMSD pattern of S3 suggests that 
(a)

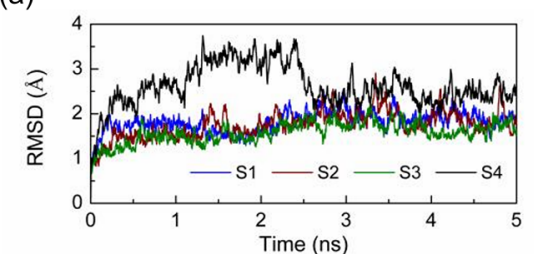

(b)

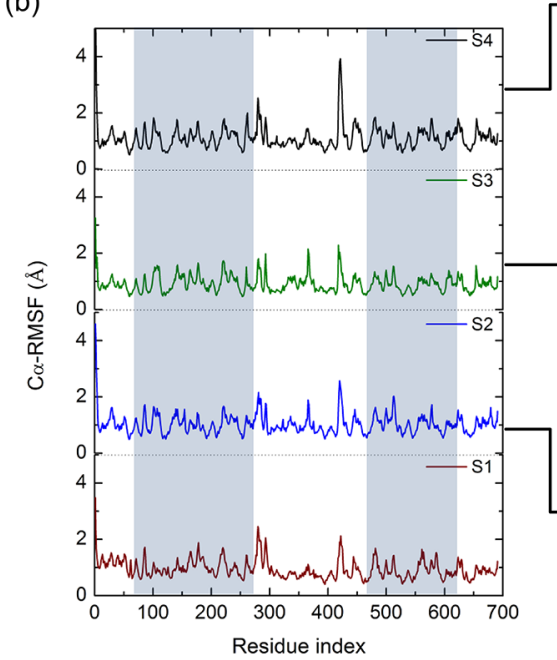

(c)

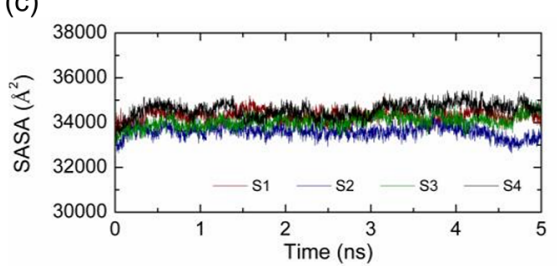

(d)

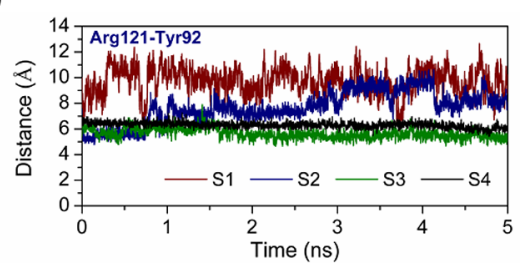

(e)

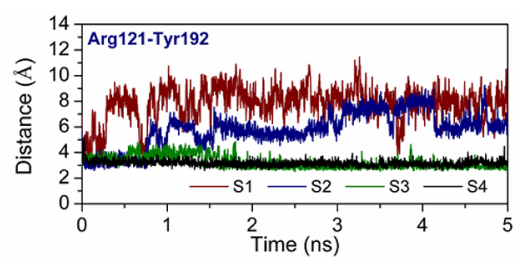

(f)

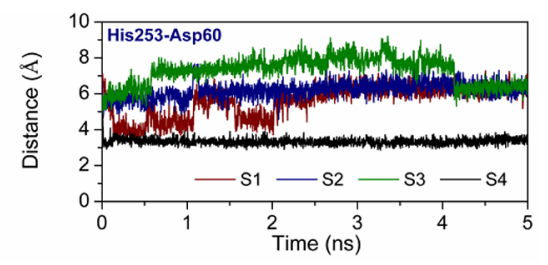

(g)

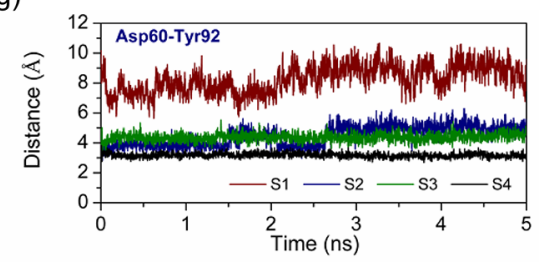

Fig. 2. Molecular dynamics simulations results. (a) RMSD; (b) RMSF of the lactoferrin backbone atoms; (c) SASA patterns and distance evolution between amino acids residues (d) Arg121 and Tyr92, (e) Arg121 and Tyr192, (f) His253 and Asp60, (g) Tyr92 and Asp60 computed along the molecular dynamics simulations of systems S1, S2, S3 and S4.

the negative charge of the carbonate ion induces a degree of stability in the closed conformation of lactoferrin. Interestingly, differences in the RMSD pattern are observed between the S1 and S2 simulations, indicating that the open form of lactoferrin fluctuates more than the closed form.

A more detailed picture of the structural behaviour and the mobility of the protein regions were obtained by computing the root mean square fluctuations (RMSF) of the atomic positions in the trajectory. Figure 2(b) displays the backbone atoms fluctuations profile of the protein in our four simulated systems.

Overall, there is a reasonable agreement between the RMSF and RMSD profiles computed for our four systems, indicating that the most noteworthy fluctuations suffer the iron-containing system (S4). The structure flexibility was also assessed through the total solvent accessible surface area (SASA) computed along the $5 \mathrm{~ns}$ trajectories of the simulated systems. The SASA plot shows a slightly different picture (see fig. 2(c)). Despite the lack of significant difference in the SASA patterns, there is an evidence of the increased flexibility of the closed conformation of apolactoferrin. This observation suggests the idea that the closed apolactoferrin system has an unstable conformation and there is a need for a local conformational rearrangement to stabilize its condition.

Distances measured between the amino acids residues from the first and second coordination shell in the simulated systems (fig. 2 (d)-(g)) show that the absence of the iron ion (system S3) induces small deviations in the protein structure. On the other hand, in the absence of the carbonate ion and $\mathrm{Fe}(\mathrm{III})$ ion (system S2), the distance increases by $2-3 \AA$. This observation confirms the significant contribution of the carbonate ion to the opening of the iron-loaded protein. This result correlates with the RMSD, RMSF and SASA evaluations.

\subsubsection{Domain opening in the absence of $\operatorname{Arg} 121$ and Arg210}

Finally, we have studied the contribution of the amino acids side-chains Arg121 and Arg210 to the conformational stability of lactoferrin. The trajectories obtained for the two systems S5 and S6 were compared with the trajectory obtained for the hololactoferrin (S4). 
(a)

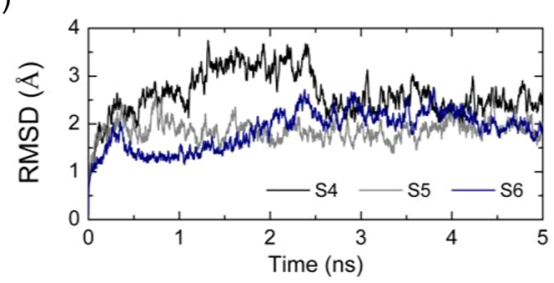

(c)

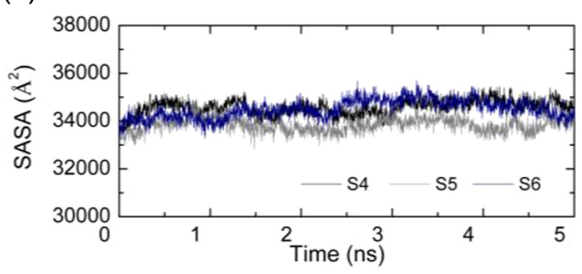

(b)

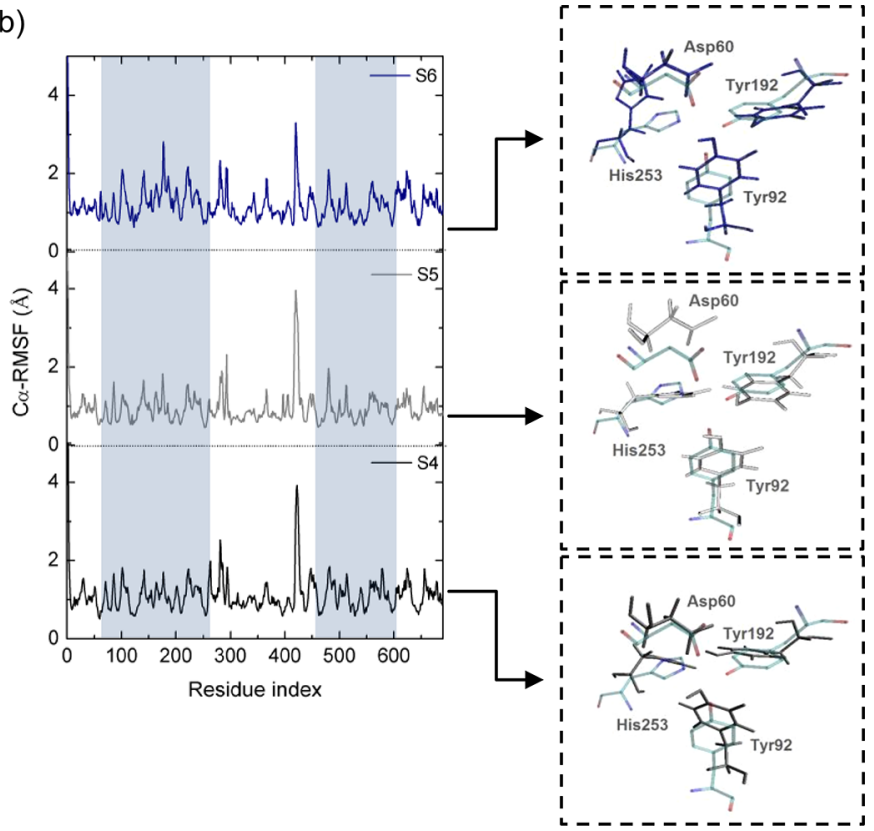

Fig. 3. Molecular dynamics simulations results. (a) RMSD, (b) RMSF of the lactoferrin backbone atoms and (c) SASA patterns computed during molecular dynamics simulations considering systems S4, S5 and S6.

The RMSD evolutions of the protein $\mathrm{C} \alpha$ atoms are shown in fig. 3(a). The absence of Arg121 and Arg210 induces conformational changes of the protein in the first $3 \mathrm{~ns}$, followed by stabilization in the last $2 \mathrm{~ns}$.

During the simulation, the RMSD pattern of the system S5 exhibits changes in the evolution if compared to system S4. This result confirms the importance of Arg121 in the conformational stability of protein. Experimental evidence shows that Arg121 stabilizes the carbonate ion in the active binding site, therefore this amino acid, indirectly, controls the rate of iron retention or release from the N-lobe of transferrins [12] and its absence destabilizes the iron binding in human lactoferrin [11]. So, the molecular dynamics calculations confirmed that Arg121, is the key amino acid in the stabilization of the Fe(III) ion in the N-lobe of human lactoferrin. The entire mechanism resembles a key-lock system. On the other hand, the RMSD pattern of system S6 exhibits the most notable changes, which can be explained by the experimental reports on lactoferrin that showed the mutation of Arg210 resulting in a local restructuration of amino acids from the N-lobe of the protein. Consequently, Lys301 changes its conformation to fill the space occupied by Arg210, interacting with two ligands from the first coordination shell Tyr92 and Tyr192 [13]. It should be noted that our results obtained from RMSD analysis of the three systems S4, S5 and S6, correlate with the changes observed in RMSF (fig. 3(b)) and SASA (fig. 3(c)) patterns.

\subsection{Open-to-closed conformation transition of human lactoferrin}

The degree of transition of lactoferrin from open to closed forms was assessed using the spectrophotometric titration method with EDTA. The binding constant of the Fe(III)EDTA complex is $10^{25.7}$ which is higher than the binding constant of lactoferrin $10^{22}$ meaning that EDTA would be an excellent chelating agent that can be used to obtain the apo-form of human lactoferrin $[3,24]$. The UV-Vis spectra obtained from spectrophotometric titration with EDTA solution are presented in fig. 4(a), the arrow on the curvature shows the decrease in absorbance at $275 \mathrm{~nm}$, which is a characteristic peak of tyrosine amino acid sidechains. We observed that in the presence of EDTA the absorbance maximum diminishes. This observation was associated with the process of iron release from the lactoferrin structure and the opening of its domains.

Structural differences between the open and closed conformation of human lactoferrin in solution were further assessed using the SANS technique to gain insights regarding the structural changes. Our rational is that a detectable difference in SANS scattering patterns between the systems Lf: $\mathrm{D}_{2} \mathrm{O}$ and Lf:EDTA: $\mathrm{D}_{2} \mathrm{O}$ would be indicative of the closed and open conformation of the protein in solution. The neutron scattering patterns are presented in fig. 4(b). Following the Guinier and inverse Fourier transform analyses, the data were subdivided to obtain the structural information of the protein within the samples.

The data in the 0.007 to $0.04 \AA^{-1} q$-range were used to estimate the radius of gyration of lactoferrin, $R_{g}=$ $26.66( \pm 1.72) \AA$, and of lactoferrin in the presence of EDTA, $R_{g}=24.67( \pm 2.02) \AA$, using the Guinier approximation. Additionally, the analysis of the pair distribution function $(p(r))$ obtained using the IFT method (fig. 4(c)) in the $0.03-0.2 \AA^{-1} q$-range gave the best estimate of the maximum dimension of the protein $D_{\max }=80( \pm 10) \AA$ and of the radius of gyration calculated in the real space of lactoferrin, $R_{g}=26.20( \pm 1.191) \AA$, and of lactoferrin in 
(a)
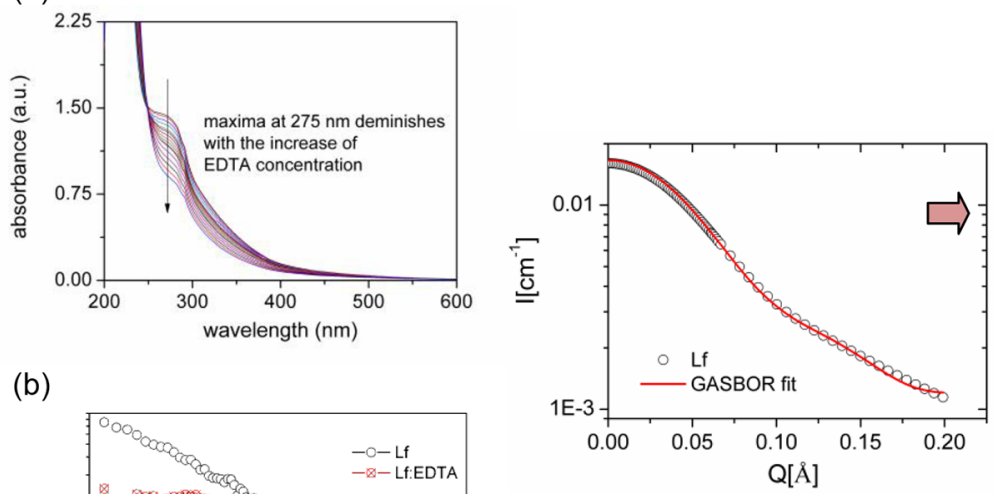

(d)

(b)
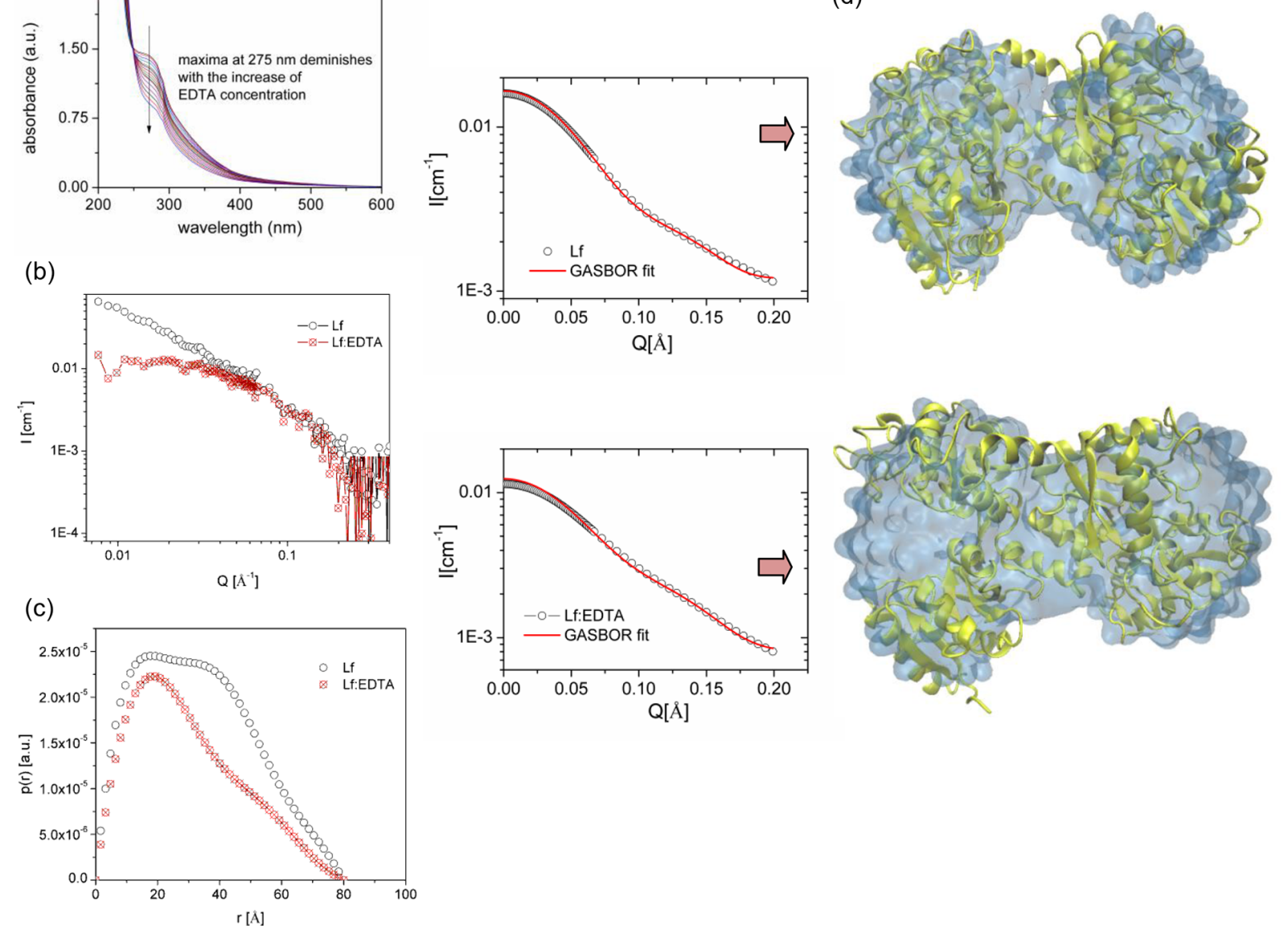

Fig. 4. Open-to-closed conformation transition of human lactoferrin study. (a) UV-Vis spectra of the titration experiment of a $2 \mathrm{mg} / \mathrm{mL}$ solution of iron-containing human lactoferrin with EDTA, at room temperature. (b) SANS curves of Lf and Lf:EDTA in HEPES buffer with $100 \% \mathrm{D}_{2} \mathrm{O}$. (c) Comparison of the $p(r)$ functions of Lf and Lf:EDTA in HEPES buffer with $100 \% \mathrm{D}_{2} \mathrm{O}$. (d) Superposition of the regularized experimental and modeled $I(q)$ curves of Lf and Lf:EDTA in HEPES buffer with $100 \%$ $\mathrm{D}_{2} \mathrm{O}$.

the presence of EDTA, $R_{g}=24.16( \pm 0.707) \AA$, which is in good agreement with the Guinier estimate.

Furthermore, the pair distribution function $p(r)$ was used to compute the low-resolution three-dimensional models assuming that the lactoferrin molecules in both systems are monomeric (P1 symmetry). Multiple runs were performed using the GASBOR program from the ATSAS package. The best low-resolution model was chosen based on the lowest value of the normalized spatial discrepancy (NSD) that is calculated using the SUPCOMB program from the ATSAS package after overlaying onto the X-ray crystallographic models (1B0L and 1LFH entries). Figure 4(d) shows the best models of lactoferrin in the presence and in the absence of EDTA as well as the fit to the scattering data. Both models present a very good resemblance with the X-ray crystallographic models for the hololactoferrin (1B0L.pdb) and the open apolactoferrin (1LFH.pdb), with an NSD of 1.3161 and 1.4540 , respectively. Our results show that the structural changes of human lactoferrin during open-to-closed conformation transition are detectable with SANS. The radius of gy- ration of apolactoferin appears to be smaller than that of the hololactoferrin, $R_{g}=24.16( \pm 0.707) \AA$ and $R_{g}=$ $26.20( \pm 1.191) \AA$, respectively. The low-resolution threedimensional models computed for the both forms of human lactoferrin in solution also show visible differences, both having a more compact conformation compared to the high-resolution structure.

\section{Conclusions}

The work presented here is dedicated to the study of the conformation stability of human lactoferrin in solution. Using molecular dynamics simulations, it is shown the importance of Arg121 and Arg210 in the conformational stability of the protein. More precisely it is underlined that Arg121, is the key amino acid in stabilization of $\mathrm{Fe}$ (III) ion in the N-lobe of human lactoferrin. SANS allowed us to detect the open-to-closed conformation transition of human lactoferrin. Our results indicate that the radius of gyration of apolactoferrin appears to be smaller 
than that of the hololactoferrin, $R_{g}=24.16( \pm 0.707) \AA$ and $R_{g}=26.20( \pm 1.191) \AA$, respectively.

The low-resolution three-dimensional models computed for both forms of human lactoferrin in solution also show visible differences, both having a more compact conformation compared to the high-resolution structure.

This work was supported by a grant framework of the Romanian Plenipotentiary at JINR-Dubna, within the JINR Theme No. 04-4-1121-2015/2020. The authors gratefully acknowledge the beamtime received at FRM-II research reactor, JCNS beamline, KWS-2 for the proposal No. 10913.

\section{Author contribution statement}

LA and RVE conceived, proposed the original idea of this study, carried out the experiments, analysed the data, interpreted the results and wrote the manuscript. AR operated the KWS-2 instrument, supervised the data acquisition and normalization of the small-angle neutron scattering data and contributed to the data treatment analysis. All authors discussed the results and contributed for the final version of the manuscript. All authors have read and approved the final manuscript.

Open Access This is an open access article distributed under the terms of the Creative Commons Attribution License (http://creativecommons.org/licenses/by/4.0), which permits unrestricted use, distribution, and reproduction in any medium, provided the original work is properly cited.

\section{References}

1. Y. Lu, N. Yeung, N. Sieracki, N.M. Marshall, Nature 460, 855 (2009).

2. E.N. Baker et al., Cell. Mol. Life Sci. 62, 2531 (2005).

3. C. Sill, BMC Biophys. 9, 1 (2016).

4. L. Anghel, Chem. J. Mold. 9, 99 (2014).

5. K. Mizutani, M. Toyoda, B. Mikami, Biochim. Biophys. Acta 1820, 203 (2012).

6. S. Teraguchi et al., Appl. Environ. Microbiol. 61, 501 (1995).

7. C. Guillén et al., Ann. Rheum Dis. 57, 309 (1998).

8. M. Spadaro et al., FASEB J. 22, 2747 (2008).

9. M. Iigo et al., Biochimie. 91, 86 (2009).

10. H.M. Habib et al., Food Chem. 141, 148 (2013).

11. H.R. Faber et al., Biochemistry 35, 14473 (1996).

12. T.E. Adams et al., J. Biol. Chem. 278, 6027 (2003).

13. N.A. Peterson et al., Biochemistry 41, 14167 (2002).

14. L. Anghel, Chem. J. Mold. 10, 70 (2015).

15. H.J.C. Berendsen et al., Comput. Phys. Commun. 91, 43 (1995).

16. S. Pronk et al., Bioinformatics 29, 845 (2013).

17. N. Guex, M.C. Peitsch, Electrophoresis 18, 2714 (1997).

18. W.L. Jorgensen et al., J. Am. Chem. Soc. 7863, 11225 (1996).

19. P.E. Mason et al., J. Am. Chem. Soc. 128, 15136 (2006).

20. W. Lin et al., Inorg. Chem. 33, 884 (1994).

21. W. Humphrey et al., J. Mol. Graph. 14, 33 (1996).

22. A. Radulescu et al., J. Vis. Exp. 118, 54639 (2016).

23. M.V. Petoukhov et al., J. Appl. Crystallogr. 45, 342 (2012).

24. R. Pakdaman, M. Petitjean, J. El Hage Chahine, Eur. J. Biochem. 254, 144 (1998). 